\title{
Acute Generalized Exanthematous Pustulosis Induced by Erlotinib (Tarceva) with Superimposed Staphylococcus aureus Skin Infection in a Pancreatic Cancer Patient: A Case Report
}

\author{
Egbert Liquete Syed Ali Revark Kammo Moiz Ali Fadi Alali \\ Hima Challa Farid Fata \\ St. Joseph Mercy Oakland-Pontiac, Auburn Hills, Mich., USA
}

\section{Key Words}

Sterile pustular rash - Surrogate marker - Acute generalized exanthematous pustulosis - Epidermal growth factor receptor - Erlotinib

\begin{abstract}
Acute generalized exanthematous pustulosis (AGEP) is a rare acute reaction that is drug induced in $90 \%$ of the cases and characterized by a widespread, sterile pustular rash. Erlotinib, a small-molecule EGFR tyrosine kinase inhibitor, has been approved by the FDA for patients with pancreatic cancer and non-small cell lung cancer. Skin rash is a well-known side effect related with all EGFR blocking agents. It has been suggested that rash could be used as a surrogate marker for response and possibly be associated with prolonged survival. We report a case of rare presentation of AGEP involving an adverse effect of erlotinib. The commonly reported adverse effects of erlotinib are mild skin eruptions. However, our case describes the rare presentation of AGEP induced by erlotinib. The estimated incidence rate of AGEP is approximately $1-5$ cases per million/year.
\end{abstract}

\section{Introduction}

Erlotinib, a small-molecule EGFR tyrosine kinase inhibitor, has been approved by the FDA for patients with pancreatic cancer and non-small cell lung cancer. Skin rash is a well-known side effect related with all EGFR blocking agents. It has been suggested that rash could be used as a surrogate marker for response and possibly be associated with 
prolonged survival [1]. In common with all other EGFR inhibitors, skin rash of a highly florid nature is the dose-limiting factor and is usually reversible. There is scant data on acute generalized exanthematous pustulosis (AGEP) with superimposed Staphylococcus aureus skin infection caused by erlotinib.

Treating pancreatic cancer, the fourth leading cause of cancer-related deaths in the United States, has always been a challenge. Gemcitabine has been the standard of care in this field since the 1990s. The addition of a second cytotoxic agent (cisplatin or oxaliplatin) has demonstrated advantage in terms of response rates and progressionfree survival, but has not achieved a significant overall survival benefit [2, 3]. A phase III randomized controlled trial by the National Cancer Institute of Canada Clinical Trials Group (NCIC-CTG) has shown a statistically significant survival benefit of gemcitabine plus erlotinib compared with gemcitabine alone [4]. Based on these data, the FDA approved erlotinib to be used with gemcitabine as a first-line treatment for advanced pancreatic cancer.

AGEP is a rare acute reaction that is drug induced in $90 \%$ of the cases [5] and characterized by a widespread, sterile pustular rash. Pustules resolve spontaneously within a few days. Although the term was used for the first time in 1980 by Beylot et al. [6], the disorder is considered to be older than this. Initially, it was mistakenly included with pustular psoriasis or classified as a hypersensitivity syndrome to anticonvulsive medications [6, 7].

\section{Case Report}

Our patient is a 63-year-old man with a past medical history of pancreatic adenocarcinoma with liver metastases. He was started on gemcitabine and Tarceva. Less than 1 week after the initiation of Tarceva, the patient developed papulopustular acneiform rash on his face (fig. 1). Over the following 3 weeks, the rash persisted despite dose adjustment of erlotinib. About 2 days prior to hospital admission, the pustular rash spread to the extremities ( $\underline{\text { fig. } 2}$ ). Erlotinib was withheld and IV steroids were given. Empiric antibiotics, vancomycin and meropenem, were started initially. Wound culture grew $S$. aureus, and nafcillin was started thereafter. A skin biopsy showed mild mixed inflammatory changes with prominent dermal hemorrhage suggestive of a drug eruption ( fig. 3 ).

On the second day of admission, the patient's symptoms of itching and pain from the erythematous rash improved. On the third and fourth day in the hospital, his rash began to improve significantly and finally disappeared. Postpustular desquamation over his upper and lower extremities was noted on the fifth day. His erythematous rash gradually subsided, and the patient was discharged on the seventh hospital day.

The patient was followed up by his oncologist and was restarted with gemcitabine which he tolerated without any side effects. In addition, he was then rechallenged with Tarceva and, surprisingly, developed another erythematous pustular rash on his face and extensor surfaces plus superimposed methicillin-sensitive $S$. aureus infection. Furthermore, the patient also developed septic arthritis for which he received IV antibiotics and recovered thereafter. He received IV steroids again, and erlotinib therapy was discontinued.

\section{Discussion}

AGEP, a member of the 'neutrophilic dermatoses' was first described by Baker and Ryan in 1968 as exanthematic pustular psoriasis [8]. The term 'pustuloses exanthématiques aiguës généralisées' (PEAG) was introduced by the French 
dermatologist C. Beylot in 1980 [6]. This pustular skin eruption, with an incidence of 1-5 per million/year, has an equal age and gender distribution. It is a self-limiting disease, with the following clinical features: (1) numerous, small non-follicular, intraepidermal or subcorneal pustules $(<5 \mathrm{~mm})$ on an erythematous background; (2) typical histopathological changes; (3) fever $\left(>38^{\circ} \mathrm{C}\right)$; (4) blood neutrophil counts $>7 \times$ $10^{9} / \mathrm{l}$, and (5) an acute evolution with spontaneous resolution of pustules within less than 15 days [8].

The AGEP validation score of the European Severe Cutaneous Adverse Reactions (EuroSCAR) study group has been used to establish the diagnosis [9]. A score between 8 and 12 for AGEP is a definitive diagnosis. In our case, the score was 11 according to the validation score of the EuroSCAR study group (table 1).

The principal differential diagnoses of AGEP consist of pustular psoriasis, Sweet's syndrome, pustular erythema multiforme, toxic epidermal necrolysis, drug rash with eosinophilia and systemic symptoms, subcorneal pustulosis (Sneddon-Wilkinson syndrome), pustular vasculitis, and bullous impetigo [9-11].

There are several theories regarding the pathogenesis of AGEP. Britschgi and Pichler [12] suggested a T cell-mediated mechanism. It can be divided into three phases. In phase I, after drug intake, antigen-presenting cells activate drug-specific $\mathrm{T}$ cells by presentation of the drug to MHC class I (for CD8+ T cells) and class II (for CD 4+ T cells) in the lymph nodes. Drugs can be covalently bound to the peptide/MHC complex or in a labile, noncovalent way. This is followed by expansion and migration of $\mathrm{T}$ cells into the dermis and epidermis.

In phase II, the infiltrating T cells release extended amounts of CXCL8, which is a neutrophil-attracting chemokine, resulting in neutrophilic inflammation. In addition to CXCL8, the T cells also induce interferon $\gamma$, interleukin 4, and interleukin 5, and these all provoke eosinophilic and neutrophilic aggregation.

Phase III is characterized by the attachment of an increasing number of neutrophils at the site of inflammation to adhesion molecules (e.g. ICAM-1) expressed on activated endothelial cells. Migration of these polymorphonuclear leukocytes along the increasing CXCL8 gradient through the dermis and the epidermis into the vesicles results in the formation of pustules.

Moreau et al. [13] proposed that AGEP is a delayed type of hypersensitivity reaction. Another possible mechanism is the production of antigen-antibody complexes induced by an infection or drug that activates the complement system, which in turn leads to neutrophil chemotaxis [8].

The typical histopathology and immunochemistry of AGEP is characterized by spongiform subcorneal and/or intraepidermal pustules, marked papillary edema (occasionally with the formation of a subepidermal blister), and polymorphous perivascular infiltrates with neutrophils and exocytosis of some eosinophils. In a minority of cases, leukocytoclastic vasculitis with fibrinoid deposition and/or focal necrosis of keratinocytes is observed.

To date, AGEP has been attributed to a variety of causes such as viral infections, Chlamydia pneumoniae infection or hypersensitivity to mercury, but the skin reaction is primarily an adverse response to drugs. Antibiotics have been implicated as the 
causative agents in $80 \%$ of the affected individuals. In our case, the offending drug was erlotinib, a small-molecule EGFR tyrosine kinase inhibitor [14]. Skin rash is a well-known side effect related with all EGFR blocking agents. It has been suggested that rash could be used as a surrogate marker for response and possibly be associated with prolonged survival [15].

Erlotinib inhibits EGFR tyrosine kinase and leads to growth arrest of keratinocyte and follicular obstruction with subsequent inflammation leading to rash development. Microbiologic stains and cultures from most cases of skin rash usually do not show an infectious cause. Superimposed secondary bacterial infection has been reported as in our case, where superimposed $S$. aureus skin infection developed. The case we report here developed a severe papulopustular rash 45 days after initiation of erlotinib, predominantly on the face but then gradually progressing to his bilateral upper extremities, chest, and lower extremities.

Due to the self-limiting course of AGEP, specific treatment is generally unnecessary. No therapy is available to prevent lesion extension and a further decline of the patient's general condition. The causative drug has to be discontinued - which we did the first day of admission - and antibiotics are not to be given unless there is superinfection of the skin lesions. Superimposed S. aureus skin infection was seen in our case, and hence nafcillin IV was given. We believe there is a chronological relationship between starting an EFGR inhibitor and the occurrence of superimposed $S$. aureus skin infection in which pustulosis is usually sterile in most of the cases. Being immunodeficient might also contribute to the development of skin infection.

In summary, we report this case in order to draw attention to and to further gain understanding of the potential adverse effects of erlotinib and to avoid unnecessary investigation and/or administration of expensive medications.

This case report also demonstrates that erlotinib (Tarceva) is becoming universally recognized as a causative agent of AGEP. However, numerous other major implications were also addressed in this case report. These include utilizing the validation score for diagnosis of AGEP, applying the appropriate treatment methods, as well as mentioning the severe infectious implications. Furthermore, this case report demonstrates the potential consequences of rechallenging patients with erlotinib, that is, additional life-threatening infectious complications as well as the unexpected and unique reoccurrence of the rare skin reaction AGEP. 
Table 1. The patient's scoring using the AGEP validation score of the EuroSCAR study group

\begin{tabular}{ll}
\hline Variable & Score \\
\hline Morphology & +2 \\
$\quad$ Pustules & +2 (typical) \\
Erythema & +2 (typical) \\
$\quad$ Distribution/pattern & +1 (typical) \\
$\quad$ Postpustular desquamation & yes \\
Course & \\
$\quad$ Mucosal involvement & 0 (no) \\
Acute onset $(<10$ days $)$ & 0 (yes) \\
Resolution $(<15$ days $)$ & 0 (yes) \\
Fever $\left(>38.7^{\circ} \mathrm{C}\right)$ & 0 (no) \\
Polymorphonuclear neutrophils $\left(>7,000 / \mathrm{mm}^{3}\right)$ & +1 (yes) \\
Histology & +3 \\
Spongiform subcorneal and/or intraepidermal pustule(s) with papillary edema & +3 \\
Total & 11 \\
\hline
\end{tabular}

$\leq 0$ = no AGEP; $1-4$ = possible AGEP; 5-7 = probable AGEP; $8-12$ = definite AGEP.

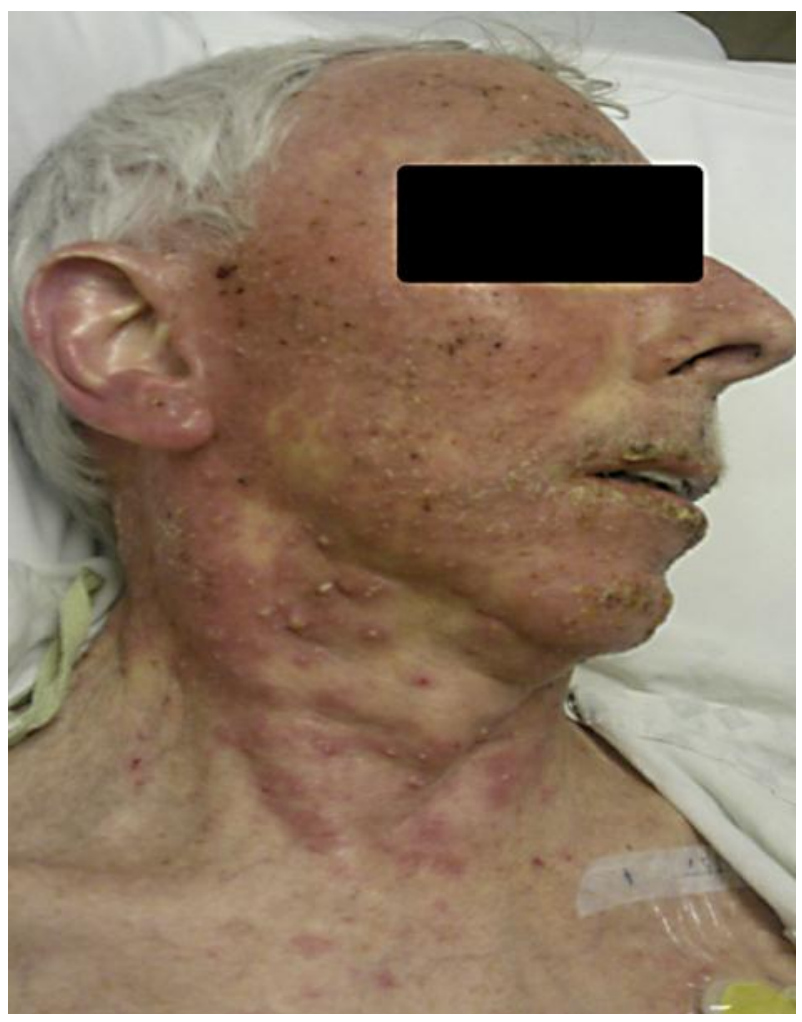

Fig. 1. Severe rash associated with erlotinib. 


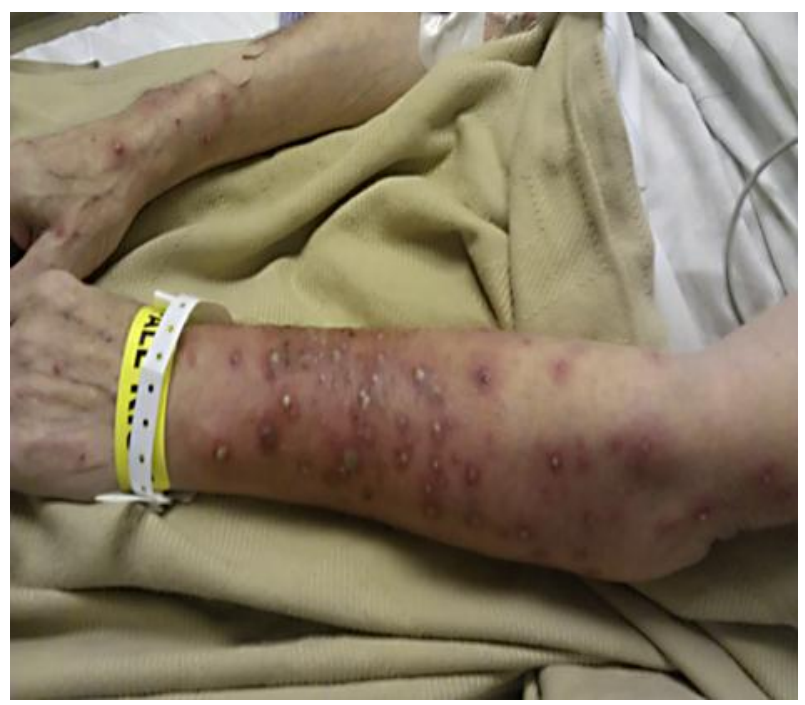

Fig. 2. Pustular rash seen on the extremities.

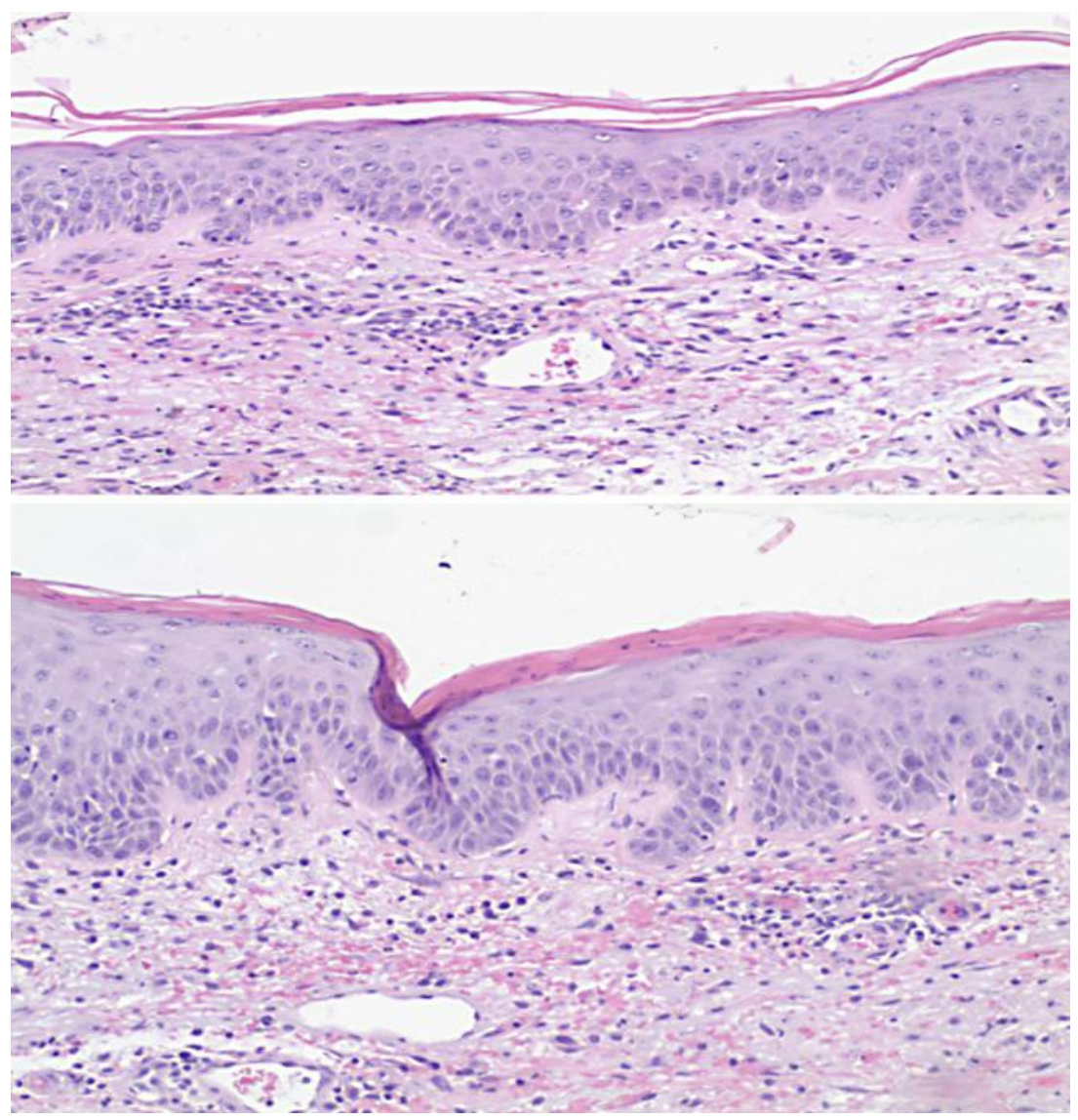

Fig. 3. Mild spongiotic change with infiltration of occasional lymphocytes. Squamous cells show some nuclear enlargement and increased mitotic activity with occasional multinucleated cells noted. There is no evidence of vasculitis. 


\section{References}

1 Perez-Soler R: Rash as a surrogate marker for efficacy of epidermal growth factor receptor inhibitors in lung cancer. Clin Lung Cancer 2006;8(suppl 1):S7-S14.

-2 Burris HA 3rd, Moore MJ, Andersen J, Green MR, Rothenberg ML, Modiano MR, et al: Improvements in survival and clinical benefit with gemcitabine as first-line therapy for patients with advanced pancreas cancer: a randomized trial. J Clin Oncol 1997;15:2403-2413.

-3 Louvet C, Labianca R, Hammel P, Lledo G, Zampino MG, André T, et al: Gemcitabine in combination with oxaliplatin compared with gemcitabine alone in locally advanced or metastatic pancreatic cancer: results of a GERCOR and GISCAD phase III trial. J Clin Oncol 2005;23:3509-3516.

4 Moore MJ, Goldstein D, Hamm J, Figer A, Hecht JR, Gallinger S, et al: Erlotinib plus gemcitabine compared with gemcitabine alone in patients with advanced pancreatic cancer: a phase III trial of the National Cancer Institute of Canada Clinical Trials Group. J Clin Oncol 2007;25:1960-1966.

$>5$ Sidoroff A, Dunant A, Viboud C, Halevy S, Bavinck JN, Naldi L, Mockenhaupt M, Fagot JP, Roujeau JC: Risk factors for acute generalized exanthematous pustulosis (AGEP) - results of a multinational case-control study (EuroSCAR). Br J Dermatol 2007;157:989-996.

6 Beylot C, Bioulac P, Doutre MS: Acute generalized exanthematic pustuloses (four cases) (in French). Ann Dermatol Venereol 1980;107:37-48.

7 Britschgi M, Steiner U, Schmid S, et al: T-cell involvement in drug-induced acute generalized exanthematous pustulosis. J Clin Invest 2001;107:1433-1441.

-8 Baker H, Ryan TJ: Generalized pustular psoriasis. A clinical and epidemiological study of 104 cases. Br J Dermatol 1968;80:771-793.

9 Guevara-Gutierrez E, Uribe-Jimenez E, Diaz-Canchola M, Tlacuilo-Parra A: Acute generalized exenthematous pustulosis: report of 12 cases and literature review. Int J Dermatol 2009;48:253-258.

10 Hall AP, Tate B: Acute generalized exenthematous pustulosis associated with oral terbinafine. Australas J Dermatol 2000;41:42-45.

11 Sidoroff A, Halevy S, Bavinck JN, Vaillant L, Roujeau JC: Acute generalized exanthematous pustulosis (AGEP) - a clinical reaction pattern. J Cutan Pathol 2001;28:113-119.

12 Britschgi M, Pichler WJ: Acute generalized exanthematous pustulosis, a clue to neutrophil-mediated inflammatory processes orchestrated by T cells. Curr Opin Allergy Clin Immunol 2002;2:325-331.

13 Moreau A, Dompmartin A, Castel B, Remond B, Leroy D: Drug-induced acute generalized exanthematous pustulosis with positive patch tests. Int J Dermatol 1995;34:263-266.

14 Rusch V, Mendelsohn J, Dmitrovsky E: The epidermal growth factor receptor and its ligands as therapeutic targets in human tumors. Cytokine Growth Factor Rev 1996;7:133-141.

15 Perez-Soler R: Rash as a surrogate marker for efficacy of epidermal growth factor receptor inhibitors in lung cancer. Clin Lung Cancer 2006;8(suppl 1):S7-S14. 